\title{
Le tiers secteur du logement dans la région métropolitaine Vienne-Bratislava
}

Recompositions d'une offre de logement abordable et fabrique métropolitaine

\section{Aurore Meyfroidt}

\section{(2) OpenEdition}

\section{Journals}

\section{Édition électronique}

URL : http://journals.openedition.org/cdg/1224

DOI : $10.4000 /$ cdg. 1224

ISSN : $2107-7266$

\section{Éditeur}

UMR 245 - CESSMA

\section{Référence électronique}

Aurore Meyfroidt, «Le tiers secteur du logement dans la région métropolitaine Vienne-Bratislava », Carnets de géographes [En ligne], 10 | 2017, mis en ligne le 30 septembre 2017, consulté le 24 septembre 2020. URL : http://journals.openedition.org/cdg/1224 ; DOI : https://doi.org/10.4000/cdg 1224

Ce document a été généré automatiquement le 24 septembre 2020

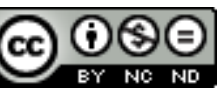

La revue Carnets de géographes est mise à disposition selon les termes de la Licence Creative Commons Attribution - Pas d'Utilisation Commerciale - Pas de Modification 4.0 International. 


\section{Le tiers secteur du logement dans la région métropolitaine Vienne- Bratislava}

Recompositions d'une offre de logement abordable et fabrique métropolitaine

\section{Aurore Meyfroidt}

1 Le Nouvel Agenda Urbain (Conférence Habitat III à Quito, octobre 2016), défendant le rôle du logement coopératif pour une urbanisation durable, questionne avec acuité l'accès au logement et le renouveau de solutions alternatives comme celles représentées par le tiers secteur du logement.

2 Cette thèse traite de ce secteur particulier de la promotion, dispersé en de nombreux sous-segments (coopératives, habitat participatif, collaboratif...), mais s'inscrivant dans l'héritage des coopératives du XIXème siècle. Le tiers secteur est hybride, empruntant aux objectifs d'utilité publique du logement social tout comme à une exigence de viabilité voire de rentabilité dans le cadre du secteur de marché. Influencé par ces deux pôles, il s'en distingue par un fonctionnement basé sur la contribution des habitants, qu'elle soit financière et/ou collective.

3 La région métropolitaine Vienne-Bratislava, souvent étudiée " par le haut » à travers la coopération transfrontalière ou la compétitivité des deux capitales à l'échelle européenne, est ici analysée par les dynamiques à l'œuvre dans la fabrique processuelle de la région, c'est-à-dire la métropolisation et la périurbanisation. Les deux capitales présentent, à leur échelle nationale respective, un héritage commun des coopératives du logement. Cet espace présentait également des pratiques mobilitaires, sous l'Empire austro-hongrois, qui avaient rapproché Vienne et Bratislava. Si aujourd'hui, suite à la transition post-communiste et à la privatisation du parc de logements municipaux et coopératifs en Slovaquie, le poids de ce segment est disparate de part et d'autre de la frontière (il assure $65 \%$ de la construction neuve à Vienne, et respectivement $49 \%$ et $39 \%$ dans les Länder voisins du Burgenland et de Basse-Autriche, contre $0,2 \%$ à Bratislava malgré des tentatives de redynamisation du secteur pour pallier la 
fragmentation socio-spatiale de cette ville post-socialiste), le questionnement fait sens en raison d'une tradition de ce tiers secteur des deux côtés de la frontière, de la proximité topographique ( 55 kilomètres entre les deux capitales) avec un phénomène émergent de périurbanisation transfrontalière, et d'une réflexion sur la diffusion des modèles urbains.

En effet, la réflexion ne concerne pas les marchés du logement mais davantage les logiques de production de ce tiers secteur, et notamment les acteurs qui le construisent : les promoteurs d'utilité publique. Il s'agit d'étudier leurs pratiques, leurs représentations d'eux-mêmes et du secteur et enfin les opérations immobilières qu'ils édifient dans cette région, de part et d'autre de la frontière nationale et des limites régionales (le logement étant une compétence des régions). Ainsi, suivant une approche de géographie régionale, nous questionnons le rôle du tiers secteur dans l'intégration de la région métropolitaine Vienne-Bratislava. Sans être dans une posture comparatiste, et au-delà d'une simple interrogation sur l'abaissement ou non de la frontière nationale, la fabrique métropolitaine que nous envisageons relève de la territorialisation des pratiques des promoteurs - y compris au-delà des frontières régionales, et des espaces produits par le tiers secteur, dans une dimension processuelle, physique et pragmatique.

5 Une méthodologie mixte a permis de répondre à ce questionnement, selon une approche à la fois structurelle et actorielle. Une base de données de 480 projets (soit près de 30000 logements) construits entre 2007 et 2014 par les promoteurs d'utilité publique de Vienne, de Basse-Autriche, du Burgenland et de Bratislava, a été confrontée à une deuxième base, rassemblant les 372 communes de la région métropolitaine, classées en cinq types selon des variables démographiques, socioéconomiques, et selon le poids du tiers secteur dans le parc de logements ( 1 : périurbain ancien et aisé à l'ouest de Vienne où le tiers secteur peine à s'implanter, $2:$ Bratislava, métropole en rattrapage, 3: espace rural sous influence urbaine, 4: métropole viennoise en croissance et où le tiers secteur se conforte dans les arrondissements périphériques, 5 : nouveaux espaces périurbains). Ces données permettent de mettre le discours des promoteurs en perspective. Des entretiens semi-directifs avec 38 acteurs institutionnels ont été complétés par un corpus de 27 entretiens avec des promoteurs d'utilité publique, et 6 entretiens avec des promoteurs privés.

6 Un premier résultat de la thèse révèle une intégration de la région par la diffusion des opérations construites dans le cadre du tiers secteur. Le tiers secteur s'inscrit dans des territoires diversifiés, ce qui illustre la dimension processuelle de la fabrique métropolitaine, en lien avec la périurbanisation vers la Basse-Autriche. Ainsi, la vision traditionnelle d'un logement abordable centré sur Vienne est remise en cause, puisque le tiers secteur se diffuse dans des tiers espaces, dans certains types de périurbain. S'il semble perdre du terrain autour de Wiener Neustadt au sud de Vienne, il se diffuse dans les espaces ruraux du Burgenland, y compris dans la frange transfrontalière. 
Figure 1. Rapport entre nouveau bâti dans le tiers secteur et structure du parc de logements
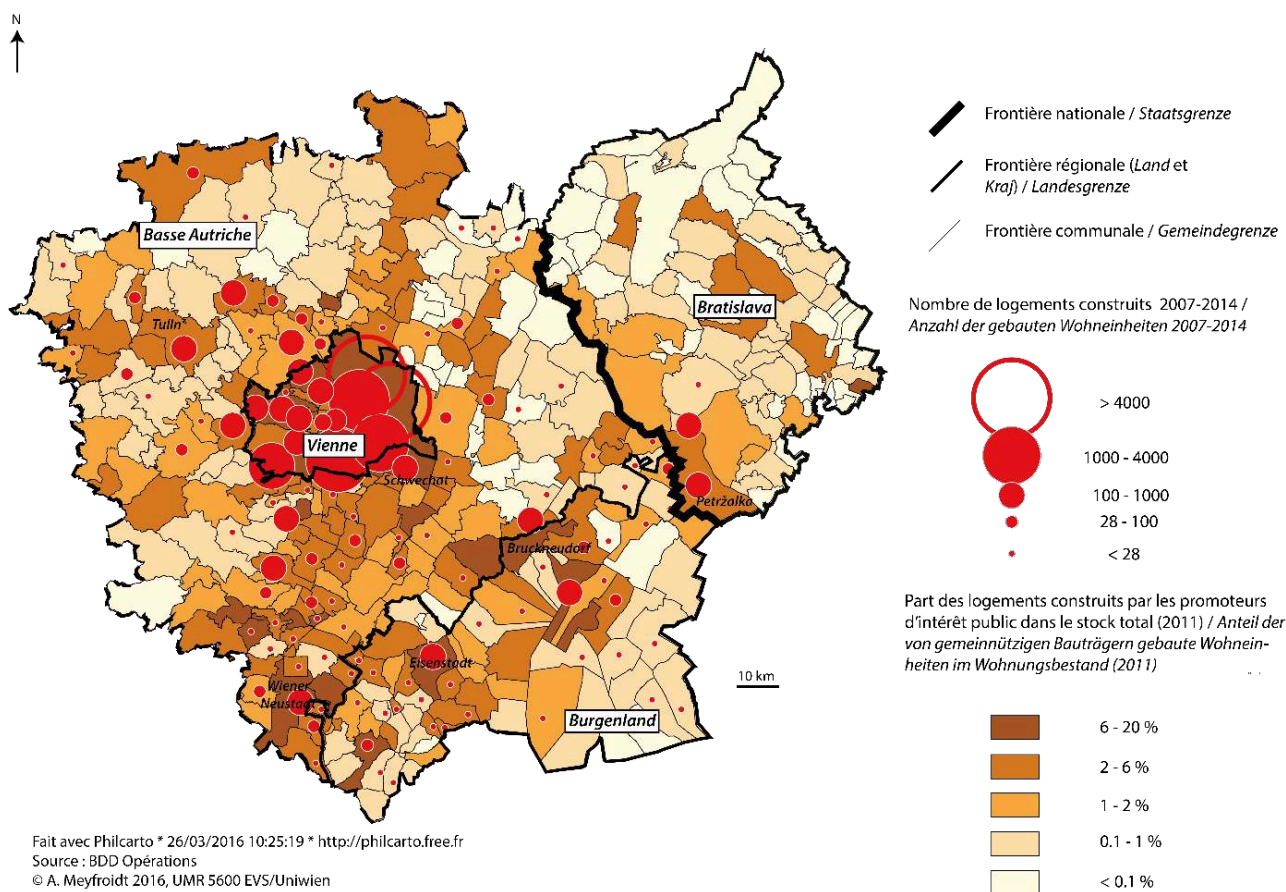

Part des logements construits par les promoteurs d'interêt public dans le stock total (2011)/Anteil der von gemeinnazizigen Bautragern ge

A. Meyfroidt 2016, UMR 5600 EVS/Uniwie

Figure 2. "Pourquoi habiter en Autriche ?" Publicité d'un promoteur d'utilité public autrichien à Bratislava

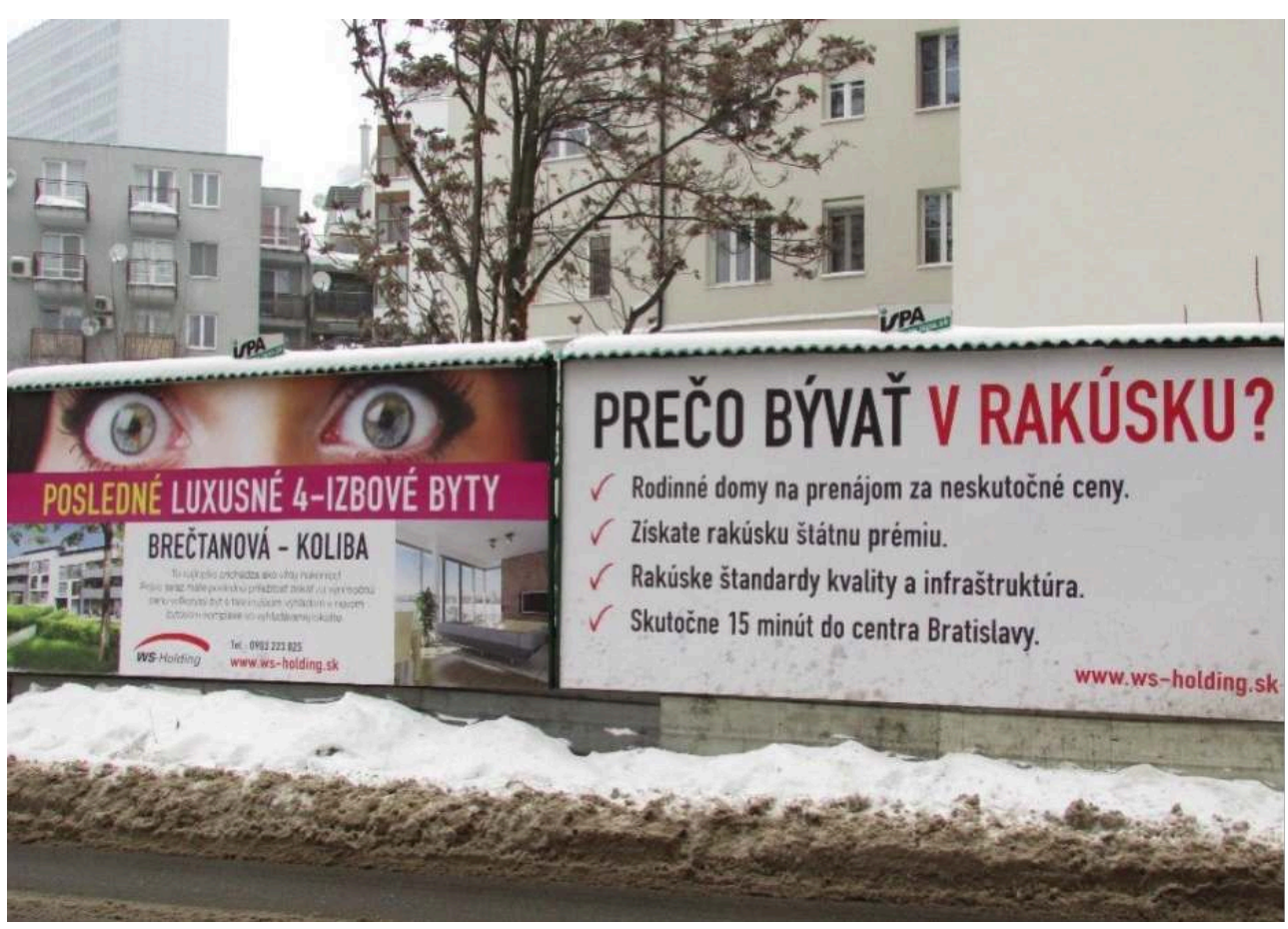

(c) AM 2013

7 En ce qui concerne la concordance entre la production résidentielle et le profil des communes, le tiers secteur est présent dans les cinq types d'espaces de la typologie. Ses formes de bâti et ses modèles de financement sont alors adaptés au profil des communes, prouvant la plasticité de ce secteur hybride : ainsi, le tiers secteur construit 
dans le périurbain ancien, rassemble majoritairement des maisons jumelées ou mitoyennes.

Enfin, la diffusion du modèle du tiers secteur autrichien, qui résiste aux tendances à la marchandisation du logement social à Vienne grâce à un fort soutien politique de la Ville-Land (ce secteur participant du rayonnement du modèle d'Etat-Providence autrichien à l'échelle européenne), se heurte cependant en Slovaquie aux structures nationales et aux héritages cognitifs post-socialistes, où le tiers secteur est associé aux coopératives d'habitat communiste, alors que de nombreux échanges ont lieu à la fois au niveau institutionnel et des promoteurs eux-mêmes (dont certains ont construits quelques opérations à Bratislava).

Figure 3. Une diffusion du modèle autrichien du tiers secteur entravée par les structures nationales

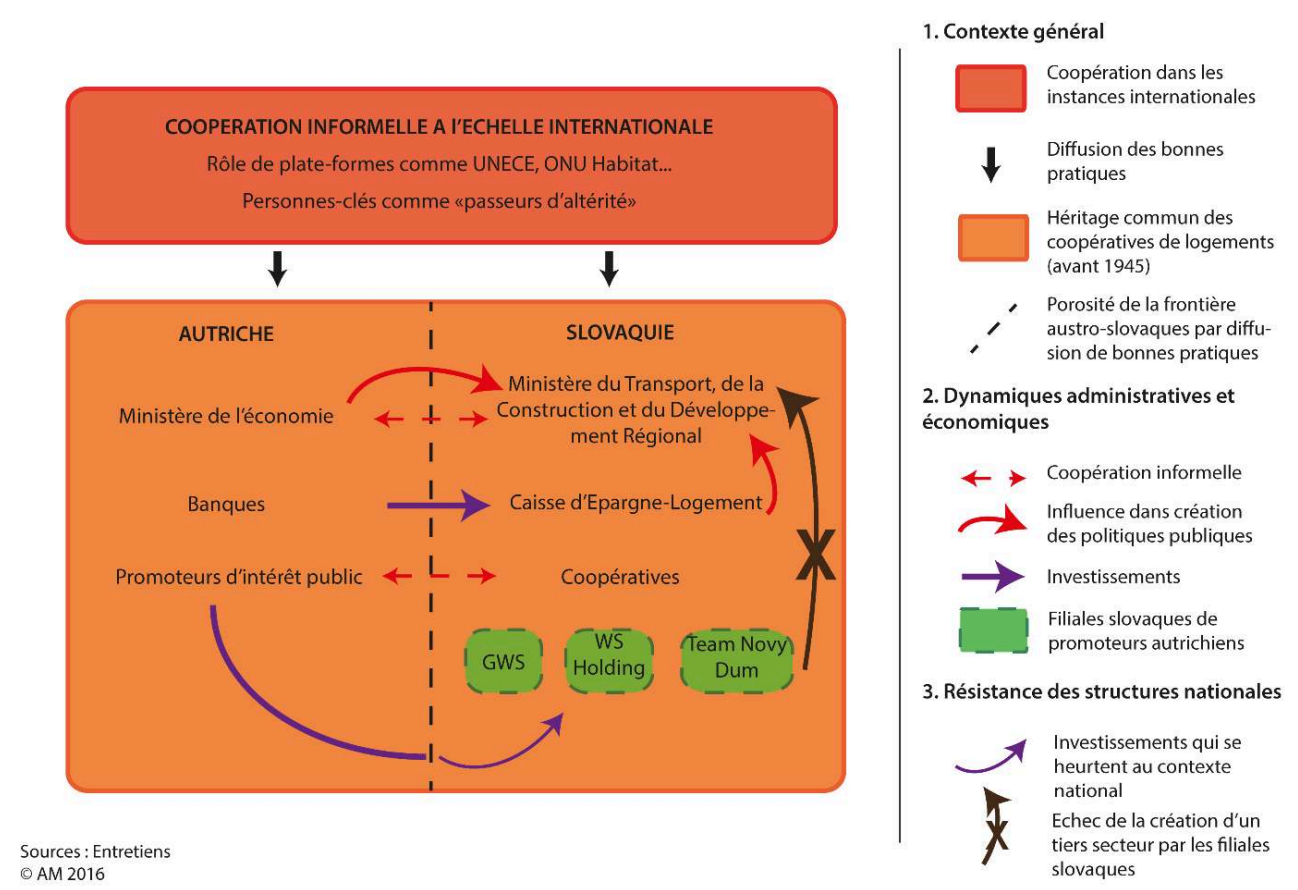

9 Finalement, le tiers secteur révèle une intégration silencieuse de la région, qui pourrait être confortée par le rôle de grands projets comme Seestadt Aspern (devant accueillir 20000 habitants d'ici 2028, à mi-chemin entre les deux capitales) et la réussite (ou non) de la recréation d'un véritable secteur locatif abordable en Slovaquie.

\section{Lien électronique si la thèse est disponible en ligne}

https://tel.archives-ouvertes.fr/tel-01419517

\section{Discipline \\ Géographie}

\section{Directeur}

Lydia Coudroy de Lille, Professeure à l'Université Lyon 2 - Lumière, Directrice de thèse / Heinz Fassmann, Professeur à l'Université de Vienne, Co-tuteur de thèse 


\section{Université}

ENS Lyon / Université de Vienne

Membres du jury de thèse, soutenue le 07 décembre 2016

Christoph Reinprecht, Professeur à l'Université de Vienne (Président du Jury)

Christian Vandermotten, Professeur à l'Université Libre de Bruxelles (Rapporteur)

Claire Lévy-Vroelant, Professeure à l'Université Paris 8 Saint-Denis (Rapporteure)

Claire Carriou, Maître de Conférences à l'Université Paris Ouest Nanterre la

Défense (Examinatrice)

\section{Situation professionnelle à l'issue de la thèse}

Post-doctorante, UMR PACTE, Université Grenoble Alpes, Chercheure associée à

l'UMR 5600 EVS

\section{Courriel}

ameyfroidt[at]gmail.com

INDEX

Thèmes : Carnets de soutenances 